\title{
Participación política juvenil en el posacuerdo: una mirada a los casos de los municipios de San Carlos y de San Francisco en el departamento de Antioquia, en Colombia*
}

\author{
Jessica Julieth Cardona Jaramillo**
}

Recibido: 5 de septiembre de 2018

Evaluado: 28 de septiembre de 2018

Aceptado: 23 de octubre de 2018

Citar como: Cardona Jaramillo, J. J. (2019). Participación política juvenil en el posacuerdo: una mirada a los casos de los municipios de San Carlos y de San Francisco en el departamento de Antioquia, en Colombia. Hallazgos, 16(31), 17-40. Doi: https://doi.org/10.15332/s1794-3841. 2019.0031 .01

\section{RESUMEN}

El presente artículo plantea un análisis comparado entre los planes de desarrollo municipales (PDM) de 2012 y de 2016, con respecto a la participación política juvenil, en territorios que fueron escenarios del conflicto interno colombiano como los casos de los municipios de San Carlos y de San Francisco en el departamento de Antioquia. A partir de este análisis, se vinculan los resultados obtenidos en las entrevistas realizadas a los funcionarios de los planteles educativos de los municipios en estudio. Al respecto, el trabajo analiza que, si bien el acuerdo final de paz ha posibilitado en lo formal la reapertura de los mecanismos legales de participación política, este ejercicio por parte de los jóvenes de estos municipios es percibido como frágil, debido a las pocas garantías que les ofrece. Se concluye que la participación política juvenil que experimentan estos municipios corresponde a formas de ejercicio "no tradicional"**** la cual es percibida por los jóvenes como el mecanismo más efectivo para hacer valer su voz en medio de la sociedad.

Palabras clave: políticas institucionales, participación política juvenil, municipios en fase de posacuerdo.

Artículo de investigación suscrito a la investigación "Impacto socioeconómico del desminado humanitario: análisis de los casos de restitución de tierras de las comunidades de San Francisco y San Carlos (Antioquia)", adscrito al semillero de investigación Seguridad, Salud y Sociedad (Sesaso), financiado por la Universidad Militar Nueva Granada, Código INV-EES-2340, Bogotá, Colombia, 2017, y dirigido por el profesor William Pachón Muñoz, coinvestigador del proyecto.

** Estudiante de último semestre del programa de Relaciones Internacionales y Estudios Políticos de la Universidad Militar Nueva Granada. Bogotá, Colombia. Correo electrónico: U0901862@unimilitar.edu.co

*** En este artículo, el ejercicio de participación política juvenil "no tradicional" corresponde a las expresiones juveniles culturales que operan como símbolo de participación política en la sociedad actual. A esto la investigadora Reguillo (2004) le ha llamado desdramatización o perfomatividad juvenil. 


\section{Youth political participation in the post-agreement: a look at the cases of the municipalities of San Carlos and San Francisco in the department of Antioquia, in Colombia}

\begin{abstract}
This article proposes a comparative analysis between the municipal development plans (PDM) of 2012 and 2016, with respect to youth political participation in territories that were scenarios of the Colombian internal conflict, such as the cases of the municipalities of San Carlos and San Francisco in the department of Antioquia. Based on this analysis, the results obtained in the interviews made to officials of the educational establishments of the municipalities under study are connected. In this regard, the paper analyzes that, although the final peace agreement has formally allowed the reopening of the legal mechanisms of political participation, this exercise by young people in these municipalities is perceived as fragile, due to the few guarantees it offers them. It is concluded that the youth political participation experienced by these municipalities corresponds to "non-traditional"*** forms of exercise, which is perceived by young people as the most effective mechanism to assert their voice in the midst of society.
\end{abstract}

Keywords: institutional policies, youth political participation, municipalities in the post-agreement phase.
Received: September 5, 2018

Evaluated: September 28, 2018.

Accepted: October 23, 2018 


\section{Participação política juvenil no pós-acordo: um olhar aos casos dos municípios de San Carlos e San Francisco no estado de Antioquia na Colômbia}

Recebido: 5 de setembro de 2018

Avaliado: 28 de setembro de 2018

Aceito: 23 de outubro de 2018

\section{Resumo}

Este artigo expõe uma análise comparada entre os planos de desenvolvimento municipais (PDM) de 2012 e de 2016, com respeito à participação política juvenil, nos territórios que foram cenários do conflito interno colombiano como os casos dos municípios de San Carlos e de San Francisco no estado de Antioquia. A partir desta análise, vinculam-se os resultados obtidos nas entrevistas realizadas aos funcionários das escolas dos municípios do estudo. Ao respeito, o trabalho analisa que se bem o acordo final de paz tem possibilitado no formal a reabertura dos mecanismos legais de participação política, este exercício por parte dos jovens destes municípios é percebido como frágil devido às poucas garantias que lhes oferece. Conclui-se que a participação política juvenil que experimentam estes municípios corresponde a formas de exercício "não tradicional"*** a qual é percebida pelos jovens como o mecanismo mais efetivo para fazer valer sua voz em meio da sociedade.

Palavras-chave: políticas institucionais, participação política juvenil, municípios em fase de pós-acordo. 


\section{INTRODUCCIÓN}

Resulta de gran trascendencia para Colombia involucrar participativamente a los jóvenes que ante la actual coyuntura son etiquetados como ciudadanos protagónicos en el posacuerdo, en especial, a los jóvenes que en sus territorios han sido afectados por el conflicto armado. Sobre ellos, recae la necesidad de llevar a cabo el cumplimiento de los acuerdos de paz, sin olvido y sin repetición de hechos violentos. Por tanto, deben existir iniciativas que motiven la participación de los jóvenes en lo político, social y cultural.

Con lo anterior, este artículo propone un análisis comparado entre los planes de desarrollo municipales (PDM) de 2012 (inicio del proceso de paz) y 2016 (inicio de la etapa de posacuerdo), con respecto a la participación política juvenil, en dos de los territorios más afectados por la violencia en el oriente antioqueño (San Carlos y San Francisco). Los PDM tienen su antecedente a partir de la Constitución Política de 1991, como se establece en el artículo 339: “todo Municipio debe elaborar sus planes de desarrollo social y económico". Allí se representan las estrategias y políticas municipales con las cuales las alcaldías buscan elevar las condiciones de vida de sus habitantes.

Ahora bien, ¿por qué los municipios de San Carlos y de San Francisco? Estos municipios vivieron un fuerte golpe de violencia a finales de la década de los noventa y principios de la primera década del siglo XXI. Producto del conflicto, la tierra quedó contaminada con minas antipersonal que causaron el desplazamiento de miles de campesinos y personas habitantes de los municipios del oriente antioqueño. Sin embargo, el proceso de consolidación del territorio, y posteriormente el proceso de paz, da apertura a la recuperación de las tierras y a la normalización de la vida en las regiones. Bajo este contexto, es imperativo conocer la historia del conflicto que atravesaron los dos municipios en estudio, con el fin de comprender el impacto que esto tiene en las políticas de desarrollo y participación política de las nuevas generaciones.

Por un lado, el municipio de San Francisco, perteneciente al oriente del departamento de Antioquia, tiene una extensión territorial de $372 \mathrm{~km}^{2}$ y poblacional de 5831 habitantes, de los cuales 2920 son población rural. Limita con los municipios de Puerto Triunfo, de San Luis, de Cocorná, de Sonsón y de Argelia (Alcaldía de San Francisco, 2012).

La historia de violencia en el municipio se empieza a reseñar desde finales de la década de los noventa. Hacia la época, llegan grupos subversivos al margen de la ley (Bloque IX de las Fuerzas Armadas Revolucionarias de Colombia [FARC] y el Frente Carlos Alirio Buitrago del Ejército de Liberación Nacional [ELN]), los cuales se enfrentan al Ejército Nacional y a las Autodefensas Unidas de Colombia (AUC) del Magdalena Medio. Los grupos armados ilegales reconocieron el territorio como fuente de riqueza para la plantación de cultivos de coca, instalación de ejércitos y campamentos en los montes, debido a la "riqueza de los suelos de la región, la lejanía y la ausencia de entidades del Estado, así como las facilidades de comunicación con el Magdalena Medio y con la zona de embalses, además de la vía que comunica a Medellín con Bogotá" (Tamayo, 2017, p. 33). 
Para 2003, el municipio quedó casi deshabitado, reduciéndose a la mitad su población total, debido a los fuertes enfrentamientos entre las guerrillas y las AUC, además de las amenazas latentes a causa de las minas antipersonal. Esta situación produjo un desplazamiento forzado hacia las principales ciudades del país.

Por otro lado, el municipio de San Carlos, también ubicado en el departamento de Antioquia, tiene una extensión territorial de $702 \mathrm{~km}^{2}$ y poblacional de 16008 hab., de los cuales 9955 son población rural. Limita al norte con los municipios de San Rafael y de San Roque, al sur con el municipio de San Luis, al oriente con los municipios de Caracolí y de Puerto Nare y al occidente con los municipios de Guatapé y de Granada (Alcaldía de San Carlos, 2012).

El municipio de San Carlos tiene una composición de la violencia muy cercana a la del municipio de San Francisco, debido a que ambos municipios están ubicados en el oriente antioqueño. Durante la década de los setenta, los ríos de los municipios aledaños a San Carlos sirvieron de base para la construcción de tres embalses, los cuales impactaron negativamente la actividad económica que estaba dedicada a la agricultura y la ganadería.

La construcción de los embalses y posteriormente de la autopista Medellín-Bogotá, durante la década de los ochenta, volcó el interés del Gobierno nacional y departamental hacia la región del oriente antioqueño, proyectando sobre esta el desarrollo de industrias tradicionales y de un sector de la agroindustria destinado a la exportación (Instituto Latinoamericano para una Sociedad y un Derecho Alternativos [ILSA], 2009).
Debido a estos cambios en el suelo, el municipio de San Carlos se configuró como territorio estratégico, para los diferentes actores armados que hacían presencia permanente, quienes se disputaban el control económico, político, social y militar del oriente antioqueño. Al igual que el municipio de San Francisco, la población fue víctima de desplazamiento forzado. En 1990, la población total era de 22999 hab. y en 2010 de 15918. Cabe mencionar que el mayor número de personas desplazadas corresponde a la zona rural.

Con los sucesos de violencia en ambos municipios, los distintos grupos sociales fueron afectados en sus vidas para siempre. Entre ellos se encontraban los niños y adolescentes que hoy son los jóvenes del posacuerdo. Durante la violencia los juegos de niños fueron cambiados por sentimientos de angustia y encerramiento a causa de los ataques y reclutamientos que llevaban a cabo los grupos guerrilleros y paramilitares. Por tanto, la posibilidad de construir una vida comunitaria fue limitada, lo cual produjo un cambio radical en la vida de los jóvenes. Tanto así que su interacción en el ámbito político va a ser reducido o escaso por temor a ser asesinados.

Ahora bien, en 2008, llegó el programa de desminado humanitario a ambos municipios, que trajo consigo la tranquilidad a los pobladores. Luego de cuatro años de desminado, se declararon los dos municipios como libre de sospecha de minas antipersonal y se impulsan los programas para la restitución de tierras y el retorno de los miles de personas desplazadas. De esta manera, se da inicio a nuevas oportunidades de desarrollo e inclusión en estas regiones. 
Bajo este contexto, el presente estudio propone analizar los PDM, que precisamente van a ser los documento que orienten el renacer de las regiones después de la época de la violencia. Para ello, la pregunta que orienta el artículo es ¿hasta qué punto han incidido los PDM de los municipios de San Francisco y de San Carlos en la participación política de los jóvenes de estos municipios, una vez suscrito el acuerdo final de paz en Colombia? Esta pregunta se desglosa en las siguientes cuatro partes para tener mayor soporte en su respuesta. La primera parte presenta la incidencia que los PDM han tenido en la participación política juvenil en los dos municipios, comparando los documentos en educación, participación y cultura de cada municipio. La segunda parte analiza la transcendencia de la implementación del punto dos del acuerdo de paz en la política de desarrollo de aquellos municipios que sufrieron el conflicto armado. Allí se menciona a las nuevas generaciones como actores fundamentales para la transformación social y política de estos territorios. La tercera parte estudia las entrevistas realizadas a los funcionarios de los planteles educativos de los municipios de San Carlos y de San Francisco para concluir que la oferta en formación superior para los jóvenes ha sido mínima, por tanto, sus expectativas de vida se proyectan en lugares distintos de su territorio natal. Por último, el artículo presenta la participación política juvenil en las políticas de desarrollo municipal, la cual ha sido marginal y poco incentivada, lo que ha impedido la consolidación de una organización juvenil.

\section{Metodología}

El presente artículo de investigación utilizó el método comparativo, el cual permitió confrontar la participación política juvenil en los PDM de los periodos de 2012 a 2015 y de 2016 a 2019. El método comparativo o el análisis comparativo es un procedimiento que se ubica entre los métodos científicos más utilizados por los investigadores de ciencias sociales. El objetivo fundamental de este método consiste en la generalización empírica y la verificación de hipótesis, por medio de la utilización de técnicas cualitativas. Para ello, fue necesaria la aplicación de una entrevista semiestructurada a funcionarios públicos de las instituciones educativas y de la Secretaría de Gobierno, claves para la recolección de fuentes primarias, así como la indagación de literatura especializada, informes y normativa, clave para la recolección de fuentes secundarias como herramienta de análisis:

- Entrevista: la entrevista realizada cuenta con un esquema semiestructurado con cinco partes que corresponden al aspecto político, económico, social, de seguridad, educación y ambiente. Adicional, cada aspecto está conformado por cuatro preguntas aproximadamente. Estos temas estuvieron divididos de esta forma para llevar una pauta o guía en el momento de realizar las preguntas.

- Población: la entrevista fue realizada a cuatro personas, una de ellas identificada como funcionario en la Secretaría de Gobierno del municipio de San Carlos y las otras tres maestros de los planteles educativos en ambos municipios del oriente antioqueño (zona urbana). 


\section{Aproximación teórica a la PARTICIPACIÓN POLÍTICA}

Dado que la mira central de este análisis estará puesta en la participación política como proyecto de abordaje hacia los jóvenes, será necesario plantear algunos parámetros que sirvan de ejes conceptuales sobre los que apoyar la lectura interpretativa de la participación.

Para empezar, el término participación política está estrechamente relacionado con la ciudadanía y con el ejercicio de la democracia. Así, para que una democracia sea legítima, se necesita la participación política, la cual se entiende como la posibilidad que tienen los ciudadanos de incidir en los acontecimientos políticos. De acuerdo con Dahl (1971/2013), los gobiernos democráticos se caracterizan, principalmente, por su capacidad de responder a las preferencias de los ciudadanos sin ningún tipo de discriminación. Para ello, el autor propone tres condiciones fundamentales con el fin de que se dé la democracia en una sociedad: primero, formular sus preferencias; segundo, manifestarlas públicamente de manera individual o colectiva entre ellos mismos y ante el Gobierno; y tercero, recibir igualdad de trato por parte de aquel. Estos aspectos a su vez requieren garantías institucionales que son libertad para formar organizaciones y asociarse a ellas, libertad de expresión, libertad de voto, elegibilidad para el servicio público, derecho de los líderes políticos a competir en busca de apoyo, diversidad de fuentes de información, elecciones libres e imparciales, instituciones que garanticen que la política del Gobierno dependa de los votos y demás formas de expresar las preferencias (Cordourier, 2015).
Con estos requisitos, se pueden hallar contrastes entre los diferentes regímenes políticos, a partir de dos dimensiones propuestas por Dahl (1971): las garantías que las instituciones brindan a sus ciudadanos para oponerse al Gobierno, es decir, la amplitud con que se facilita el debate político, y la facultad para participar en el control y en la discusión de la política del Gobierno. Bajo esta premisa, la participación queda reducida a la selección de un cuerpo de representantes a través del ejercicio del voto. Sin embargo, el acercamiento de la teoría dahliana a la participación permite desarrollar este concepto en un campo más amplio. Es decir, cuando se analizan las garantías institucionales, se reconoce una serie de atributos que permiten la participación por diferentes medios que no necesariamente se encuentran relacionados con las elecciones. Desde el mismo acto de formular las preferencias y de manifestarse de manera pública, los ciudadanos están participando. En ese sentido, participar es "tener voz en un sistema de debate público" (Dahl, 1971/2013).

Por tanto, el sufragio es tan solo una de las herramientas con las que cuentan los ciudadanos para participar en una democracia. Los clásicos autores Verba y Nie (1972, p. 2) advierten que la definición de participación política debe incluir algo más que el comportamiento relacionado con los partidos políticos y las elecciones. De ahí que sea imprescindible añadir una participación no convencional que se caracteriza por el uso de otros medios distintos del voto para hacer valer su voz (marchas, protestas, huelgas, movimientos de resistencia y demás expresiones políticas no electorales).

Autores como Booth y Seligson ofrecen una definición más amplia del término 
participación política, entendiéndolo como el esfuerzo por influir en la distribución de bienes públicos, los cuales son provistos por el Gobierno o la comunidad a través del gasto. Por ende, es también considerada participación política las actividades desarrolladas por la ciudadanía en su entorno comunitario, como el barrio, en el que inciden en la distribución de un bien público (mejora de calles y parques, o rutas hacia zonas rurales) (Booth y Seligson, 1978, citados por Somuano, 2005). De este modo, la participación es acción e intención, ya que, si no se manifiesta el deseo con acciones, no hay participación política. Esta definición, al ser más amplia, implica la inclusión de la participación ciudadana dentro del tema político.

Bajo esta línea de pensamiento, es imprescindible estudiar las comunidades y los entornos sociales en los que las colectividades se reúnen para debatir sus problemas y plantear soluciones. En dichos entornos, se ejerce la participación de manera natural, fuera del ámbito de gobierno formal o convencional. Con lo anterior, se entiende que lo político ha sufrido una transformación en tres aspectos fundamentales: "primero, la deslegitimación de la política tradicional, segundo, el desplazamiento del Estado como eje central de lo político (nuevas formas de asumir lo público), y tercero, la aplicación y reivindicación de nuevas formas políticas o prácticas alternativas" (Varón, 2014, p. 120). Todo ello conlleva la construcción de nuevas formas de ciudadanía, las cuales se caracterizan por acciones impregnadas de símbolos y representaciones de tipo cultural.
La ciudadanía entonces pasa a desempeñar un papel imperante en el reconocimiento de la participación juvenil. Los jóvenes actualmente no tienen sentido de pertenencia con los territorios y con las estructuras sociales establecidas. Por tanto, identificar cómo la juventud se apropia del mundo permite establecer parámetros de participación dentro de la sociedad. Según Marshall, la ciudadanía corresponde al "status asignado a todos aquellos que son miembros plenos de una comunidad. Todos los que posean dicho status son iguales con respecto a derechos y deberes" (1998, p. 149). De ahí que la ciudadanía se relacione directamente con los intereses colectivos sobre el bien general, ya que considera los bienes públicos a cambio de una protección pública en sus derechos. Si todos son considerados iguales ante la ley, van a poder ser parte del poder político.

Esta definición reduce el concepto de ciudadanía a su relación con el Estado, sin embargo, existen otros conceptos teóricos que se acercan a la idea de ciudadanía, apropiada para el análisis de este artículo. Esta ciudadanía se desarrolla a través de la participación misma del individuo en su entorno social, es decir, se construye en medio de una convivencia social. Para Reguillo (2003), la ciudadanía es un diálogo entre los derechos sociales, políticos y culturales por medio de las experiencias y la participación del individuo con el mundo. Allí los espacios culturales adquieren un valor determinante para los grupos ignorados en las restricciones de otros modelos de ciudadanía. Por esto, los jóvenes van a tener la posibilidad de dimensionar la vida y su relación con la política desde la óptica de la ciudadanía cultural. 


\section{LA PARTICIPACIÓN POLÍTICA JUVENIL DETERMINADA POR LOS PDM}

Los PDM, entendidos como la hoja de ruta de las regiones para crear estrategias y políticas orientadas a elevar las condiciones de vida de sus habitantes, tienen su antecedente en la Ley 152/1994, de 15 de julio. A partir de esta ley orgánica, el país abrió la oportunidad para que las regiones pudieran tener autonomía en temas relacionados con el desarrollo social, económico, y la gestión ambiental. Con el paso del tiempo, se ha reforzado su contenido llegando a implementarse políticas direccionadas a los diferentes sectores de la sociedad, y por eso hoy los jóvenes toman gran relevancia en la lucha por sus derechos. De ahí que sea imperativo analizar los PDM, en los cuales se proponen políticas que van a determinar la participación juvenil, analizando importantes elementos de desarrollo, como la educación, la cultura y los programas de participación.

En la búsqueda de construir el sentido a la sociedad, la educación se convierte en el sistema imperante de la historia moderna; es a través de esta que se produce la sociedad, la cual genera sentido de pertenencia social a los actores que la componen diferenciados por sus condiciones económicas o socioculturales. De esta forma, promueve la inclusión de todos los integrantes de la sociedad en un sistema educativo universal que es impulsado por políticas estatales. Por tanto, si los jóvenes se educan, "se activan las capacidades críticas y reflexivas, lo que incide en el fortalecimiento de las prestancias cognitivas e intelectuales, a través de procesos de enseñanza-aprendizaje construidos deliberadamente con ese objetivo" (Echeverría, 2013, p. 29).
Se entiende que la educación es una política pública, que actúa como principal instrumento participativo del que se dota la sociedad moderna para darse sentido a sí misma. Su influencia es tan determinante que incide en los otros sistemas de reproducción social, económico y político. De ahí que se visualice a los colegios como centros de formación valiosa para "educar a los jóvenes como ciudadanos, lo que implicaría el reconocimiento explícito de la escuela como escenario de formación política" (Díaz, 2011, p. 26).

En este sentido, la educación es el principal elemento de análisis, debido a su repercusión en los demás aspectos sociales de una persona. Entendiendo que es necesario fortalecer los vínculos entre el sistema escolar y la sociedad, al igual que los niveles de cobertura y calidad educativa, ya que estos ajustes son imperantes para activar las capacidades críticas y reflexivas de los jóvenes. Por esto, en el periodo de 2012 a 2015, la educación tuvo gran resonancia en los planes de desarrollo. A causa de la seguridad democrática y la disminución de la violencia en los municipios de San Carlos y de San Francisco, estos experimentaron un elevado número de estudiantes, para lo cual fue necesaria la apertura de nuevas instalaciones educativas. Sin embargo, dicho incremento denota que no son suficientes los recursos económicos para suplir las necesidades en lo que tiene que ver con infraestructura, dotación, alimentación escolar, atención psicosocial, entre otros, haciéndose precisa la cofinanciación de más del 70 \% de los recursos para suplir las necesidades (Alcaldía de San Carlos, 2012).

El municipio de San Francisco ha venido mejorando su calidad educativa lentamente. En las pruebas Saber de 2014, la Institución 
Educativa San Francisco obtuvo la calificación $\mathrm{B}$ a nivel nacional (Coordinación de Educación Municipal San Francisco, 2015). Además, el municipio presenta una cobertura neta de educación media del $42,7 \%$, muy similar a las coberturas departamental y regional del 41,8 y del $42,1 \%$, respectivamente. El resultado esperado para 2018 será mantener este promedio obtenido durante 2014 (Alcaldía de San Francisco, 2016). Según lo relata Azarías Cuesta, rector de la Institución Educativa San Francisco, “después de haber pasado el trago amargo de la violencia, queda levantar el municipio con educación y la mejor forma es mejorando la calidad educativa para que los muchachos vean nuevas oportunidades y puedan mejorar su calidad de vida". Por esto, la Alcaldía municipal trabaja fuertemente en las políticas educativas de cobertura y calidad.

Hoy la institución está ubicada en el puesto 11 de a 1050 instituciones educativas del departamento de Antioquia. Ya hemos sacado dos pilos paga y en 2018 el objetivo es obtener becas que ayuden económicamente a los jóvenes que están por terminar sus estudios de bachillerato, ya que la institución ha entregado a la sociedad muy buenos estudiantes que se están quedando por fuera del sistema de educación superior por falta de oportunidades. (A. Cuesta, comunicación personal, 18 de noviembre de 2017)

Por otro lado, la educación en el municipio de San Carlos se caracteriza por su bajo rendimiento. Según las pruebas Saber 11 de 2014, cinco establecimientos fueron evaluados, dos de los cuales quedaron en clasificación C y tres en D (en la que D y C son las categorías más bajas) (Secretaría de
Educación San Carlos, 2011). Estos problemas principalmente obedecen a la dificultad financiera que presenta el municipio para atender el sector educativo, ya que la demanda en los colegios ha aumentado, debido al alto nivel de jóvenes retornados después de haber disminuido el conflicto armado y haberse declarado el municipio como libre de sospecha de minas antipersonal.

En cuanto a cobertura, el municipio presenta un déficit bastante alto comparado con el departamento y la región. El porcentaje en cobertura neta de educación media para 2014 correspondió al 38,6 \%, mientras que el departamento presenta el 43,8 \% y la región el $43,7 \%$ de cobertura. La meta que se propuso la Alcaldía para 2018 será del 43,3 \%, lo que se traduce en un alto esfuerzo de cierre de brecha, comparándolo con la meta propuesta (Alcaldía de San Carlos, 2016).

Los datos mencionados dan cuenta de las falencias en la educación de los dos municipios del oriente antioqueño, tras lo cual se concluye que la educación que reciben los jóvenes en estos municipios tiene deficiencias, las cuales se están viendo reflejas en los resultados de las pruebas estatales y, en ese sentido, en sus capacidades críticas y reflexivas que los jóvenes deben poseer para enfrentarse a la sociedad al finalizar su etapa escolar.

Ahora bien, en el periodo de 2016 a 2019, los problemas en la educación de ambos municipios son reconocidos, y así se crean estrategias para mejorar la cobertura educativa y los procesos de apertura a la educación superior, mediante apoyos económicos a los egresados y convenios interinstitucionales con instituciones de educación superior en busca de la tecnificación y profesionalización 
(Alcaldía de San Carlos, 2016). Según el rector de la Institución Educativa Joaquín Cárdenas Gómez, el municipio de San Carlos en temas educativos ha presentado mejoras en las oportunidades para los estudiantes egresados de bachillerato:

Existe una nueva orientación académica que busca encaminar a los chicos de grado décimo y once en una formación universitaria con la ayuda del SENA [Servicio Nacional de Aprendizaje] y otras instituciones educativas que están llegando al municipio; sin embrago, este proceso es lento. Por ejemplo, en 2013, se gestionó un encuentro de egresados donde se dio a conocer que solo el $2 \%$ de los que asistieron tenían educación superior. (J. Sánchez, cominucación personal, 17 de noviembre de 2017)

Las políticas en educación evidencian que existe una voluntad por parte de las entidades municipales para mejorar la calidad. Sin embargo, el proceso que se lleva a cabo para su implementación y divulgación es lento, lo que impide un progreso visible. Además, las metas propuestas por ambas alcaldías siguen estando muy por debajo de una calidad aceptable o de un nivel de cobertura bueno. Esto mantiene un sustancial retraso en la generación de outputs específicos, que se vinculan al sistema educativo de manera más estratégica, con los demás ámbitos de construcción de sociedad y de participación ciudadana (Echeverría, 2013). Es decir, los estudiantes de ambos municipios están saliendo con fallas en sus habilidades para enfrentar el sector social, económico y político. Sin mencionar que más de la mitad de los estudiantes de educación media, que corresponde a jóvenes en su mayoría, están por fuera del sistema educativo, lo cual permite deducir que la educación que reciben los jóvenes en estos municipios está afectando su capacidad reflexiva y crítica para ser parte de procesos de participación ciudadana. Y no es precisamente la educación de calidad que pregonan las entidades estatales en sus políticas públicas.

Otro punto dentro del PDM es la cultura desde la que los jóvenes expresen a la sociedad sus expectativas, inconformidades y proyectos, e incluso va a permitir que en espacios culturales ellos y ellas piensen en la educación como proceso determinante para la construcción de la ciudadanía y su proyecto de vida.

En el caso del municipio de San Francisco, el sector cultural se encuentra rezagado, por falta de apoyo en temas de memoria histórica y compromiso del sector social y juvenil. No obstante, los pocos esfuerzos se desarrollan en la casa de la cultura, que es el espacio de encuentro de los jóvenes donde expresan colectivamente su relación con lo público. Desde allí, se inició en 2012 el programa de jóvenes para impulsar la creación de la política pública juvenil, en la cual se elegía el consejo municipal de juventud (CMJ). Sin embargo, por falta de mantenimiento en la estructura y discontinuidad en los programas, el CMJ sufre un gran retraso en cuanto a la representación juvenil en el municipio de San Francisco. Por otro lado, la Alcaldía por medio del PDM creó programas en coordinación con Colombia Joven y la Secretaría de Protección Social fortaleciendo la participación juvenil (Alcaldía de San Francisco, 2016).

Ahora bien, en el municipio de San Carlos, los esfuerzos y programas de la Alcaldía han sido mayores en comparación con 
el municipio de San Francisco. En 2010, se adopta el Plan Municipal de Juventud, dentro del cual se crea el CMJ. La apertura del CMJ abrió un amplio panorama para la población joven del municipio de San Carlos, lo cual posibilitó un diálogo directo y asertivo con la población juvenil, organizaciones gubernamentales y no gubernamentales, y la gestión para la consecución de una casa de la juventud, que surge de la necesidad de generar un espacio para el desarrollo de procesos coherentes y sostenibles con y para la juventud, en la reconciliación y la construcción colectiva de tejido social como estrategia para la no repetición del conflicto armado y de paz, a través del reconocimiento de sus derechos y responsabilidades (Alcaldía de San Carlos, 2012).

Por tanto, como se ha evidenciado en los PDM de ambos municipios, los temas de participación se han venido desarrollando a través de la cultura y con mayor fuerza en el municipio de San Carlos, donde la juventud recibe apoyo directo de la Alcaldía por medio de la Casa de la Juventud y de la Cultura. Allí los jóvenes tienen un acercamiento más directo y personal a los problemas que los aquejan. Hader García, secretario de Gobierno de la Alcaldía de San Carlos, comentaba que

los jóvenes se reúnen en la Casa de la Cultura y de allí han salido grandes proyectos en relación con la memoria histórica, lo cual tiene que ver con el tema emocional, que parte y pasa por la superación del miedo, por perdonarme y perdonar, a través del programa de murales. En muchas esquinas del municipio, está quedando plasmada la historia, para mí eso es un tema educativo, que pasa por el aula de clase y llega a las aulas de la calle del municipio, esto es un ejercicio pedagógico de orden local donde los jóvenes están expresando sus realidades de manera espontánea. (comunicación personal, 17 de noviembre de 2017)

Este nuevo espacio para los jóvenes fortalece la participación en los asuntos públicos del municipio y abre la posibilidad de desarrollar programas culturales.

Además, desde la Casa de la Cultura, los jóvenes han fortalecido sus capacidades de liderazgo y habilidades para la vida, por medio de la escuela de liderazgo juvenil Conducción Democrática de Procesos Organizativos (Codepror). El proyecto hace parte de la iniciativa municipal de 2012 para incrementar la participación de los jóvenes en espacios para la formación comunitaria y la asociatividad juvenil, vinculando a los jóvenes formados como promotores del desarrollo sostenible y sustentable.

Estos programas han surgido a partir de los PDM, y se vienen desarrollando en los últimos dos periodos de gobierno municipal. Allí, se evidencia que la participación juvenil ha empezado a tomar forma dentro de las agendas de políticas locales. Por eso, hoy se puede hablar de ciudadanía juvenil y participación política, entendiendo que son formas no tradicionales de manifestación social y política.

\section{El PUNTO dos DEL ACUERdo DE PAZ SOBRE PARTICIPACIÓN POLÍTICA}

Por más de medio siglo, el país vivió un profundo enfrentamiento armado con grupos guerrilleros insurgentes, con los cuales 
se negoció una propuesta de paz. El conflicto ha llegado hasta tal punto de que el diálogo es la solución más sensata para todas las partes. Fruto de esta reflexión, en 2016, se firmó el "Acuerdo final para la terminación del conflicto y la construcción de una paz estable y duradera", entre el Gobierno y la Fuerzas Armadas Revolucionarias de Colombia (FARC). Dicho acuerdo pone fin al enorme sufrimiento que ha causado el conflicto, brindando la oportunidad a los millones de colombianos víctimas de vivir una vida próspera y en paz. Por primera vez, se piensa en las víctimas como los más afectados, dando cumplimiento a programas que reconocen y tienen en cuenta sus necesidades, características y particularidades económicas, culturales y sociales. Además, se distingue la participación ciudadana como fundamento del acuerdo, ya que, a través de esta, se hará seguimiento a los programas en los territorios.

El acuerdo de paz contiene seis puntos esenciales para la terminación del conflicto, de los cuales el presente artículo tiene total atención en el punto dos sobre "Participación política: apertura democrática para construir la paz". A partir de este punto, se analiza la trascendencia de su implementación en las políticas de desarrollo de los municipios de San Carlos y de San Francis$\mathrm{co}$, que fueron territorios afectados por el conflicto armado. Esta guerra marcó fuertemente el desarrollo de las regiones, afectando directamente la calidad de vida de las personas, en especial a un gran número de víctimas, que años después regresan a su territorio para reclamar la tierra que legítimamente les pertenece.
Ante estas transformaciones, los municipios se ven en la necesidad de atender a las exigencias de la comunidad, brindando mayor atención en la cobertura de servicios públicos, educación, salud, programas culturales, deportivos, ambientales, etc. El involucramiento de la sociedad en el debate con las instituciones para atender a sus necesidades permitió que se crearan por primera vez programas que estuvieran enfocados en las demandas de los diferentes grupos de la sociedad, con especial atención en las víctimas. Por esto, los PDM actuales tienen como propósito la inclusión social, que se desarrolla a través de la participación de la comunidad en la construcción de propuestas conjuntas en pro de un mejor bienestar para todos.

Por medio de los acuerdos pactados, se crean políticas que afectan directamente el desarrollo de las regiones, las cuales están en pleno proceso de construcción de paz. Parte de esto, requiere el fortalecimiento de la democracia, en la que nuevas voces se unan al escenario político para incluir a todos los sectores de la sociedad y permitir un control eficiente en torno a lo público. La participación es entonces completamente imperante dentro de la construcción y consolidación de la paz. Es asunto de la sociedad en su conjunto que todas las personas sin distinción participen en los distintos escenarios públicos.

Desde el punto dos del acuerdo de paz, se hace un llamado a cumplir con los deberes de participación ciudadana y se asegura que el Estado garantizará los movimientos y las organizaciones sociales en los territorios. El acuerdo establece que el Gobierno nacional elaborará un proyecto de ley de garantías y promoción de la participación ciudadana, 
en la cual se apoya, "mediante asistencia legal y técnica, la creación y el fortalecimiento de las organizaciones y movimientos sociales. Sin perjuicio del principio de igualdad, se apoyará con medidas extraordinarias a las organizaciones de mujeres, de jóvenes y de grupos históricamente discriminados" ("Acuerdo final para la terminación del conflicto y la construcción de una paz estable y duradera", 2016, p. 43). Esta promoción de participación trasciende en la política de desarrollo municipal y reconoce que los jóvenes han sido históricamente discriminados. Por tanto, es momento de que en medio del posacuerdo se reconozcan a las juventudes como un grupo heterogéneo y supremamente importante para la construcción y consolidación de la paz.

Sin embargo, el trabajo que se debe llevar a cabo con las juventudes va mucho más allá del reconocimiento de la diferencia y de una ciudadanía juvenil, puesto que la historia que enmarca a la generación actual fue trastocada por una violencia altamente política.

Organizarse, participar políticamente, criticar y plantear proyectos alternativos representó un peligro, una "causa" de la violencia, y llevó a que muchos adultos y en especial jóvenes no quieran saber nada de organización ni de política, pues es mejor "no estar metido en nada, mantenerse aislado y no saber de nada". En el contexto de la guerra, la política se convierte en un ámbito de peligro y de muerte, de contradicciones y de conflictos, hasta el punto de que decir "no estamos en política" es hoy una fuente de virtud y garantía de transparencia (Grupo de Memoria Histórica, 2011).

Ahora bien, la participación ciudadana más importante se concentra en las juntas de acción comunal (JAC), que para cada municipio se compone de la siguiente manera: para 2010, en el municipio de San Carlos, se encontraron registradas 80 JAC y 21 organizaciones sociales activas conformadas por diferentes grupos que hacen parte de la población víctima del desplazamiento forzado: mujeres cabeza de hogar, campesinos, afrocolombianos, productores y consumidores del municipio, hogares familiares, discapacitados y adultos mayores (Alcaldía de San Carlos, 2012). En el caso del municipio de San Francisco, hay legalmente constituidas 31 JAC de las cuales 27 están en el área rural y 4 en las cabeceras urbanas (Alcaldía de San Francisco, 2016).

A pesar de que la comunidad se puede manifestar a través de estos grupos, en la práctica las alcaldías no han dimensionado la importancia de la acción comunal y participación en el desarrollo. Así es que los grupos juveniles no se encuentran registrados como organizaciones dentro de los municipios. Los jóvenes son un sector muy importante de la sociedad y cuentan con sus propias realidades que no están siendo atendidas de manera efectiva por las entidades municipales. Esto quiere decir que, sumado a los problemas juveniles como la deserción escolar, los embarazos a temprana edad, el consumo de sustancias psicoactivas, se debe adicionar la inclusión social y el trabajo con víctimas juveniles, por medio de programas que se enfoquen en la participación política juvenil. Según Dastres (2003), con el tiempo, esta participación desarrolla una generación de conciencia crítica y propositiva en los ciudadanos, en la que progresivamente estos van adquiriendo mayor relevancia y protagonismo, individual o colectivamente dentro de la sociedad. Abrir el espacio 
de reconocimiento para los jóvenes como agentes de cambio en territorios históricamente conflictivos contribuirá en la construcción de la paz.

Por otra parte, en el acuerdo de paz, se establece que la participación ciudadana también se puede llevar a cabo a través de los medios de comunicación. Los medios de comunicación comunitarios, institucionales y regionales deben contribuir a la participación ciudadana y en especial a promover valores cívicos, el reconocimiento de las diferentes identidades étnicas y culturales, la igualdad de oportunidades entre hombres y mujeres, la inclusión política y social, la integración nacional y en general el fortalecimiento de la democracia. La participación ciudadana en los medios comunitarios contribuye, además, a la construcción de una cultura democrática basada en los principios de libertad, dignidad y pertenencia, y a fortalecer las comunidades con lazos de vecindad o colaboración mutuos ("Acuerdo final para la terminación del conflicto y la construcción de una paz estable y duradera", 2016).

En este caso, los medios de comunicación a los que los estudiantes están expuestos son el periódico escolar y la emisora. Sin embargo, el trabajo que se resalta en ambos municipios es el arte callejero, por medio del cual existen expresiones que simbolizan el contexto social en el que viven las regiones.

Finalmente, la participación política juvenil se constituye en un elemento central para la construcción de la paz. Son las nuevas generaciones actores fundamentales para la transformación social y política de estos municipios. No se trata de excluir a los jóvenes por su estigmatización de apáticos e indiferentes, sino, al contrario, de valorar la diferencia, como sujetos capaces de actuar con otros en el ejercicio pleno de la ciudadanía. En ese sentido, aunque la sociedad haya delegado los colegios como los encargados de construir la ciudadanía, es necesario fortalecer otros escenarios de socialización, como la familia, los grupos sociales, los medios de comunicación, los mismos partidos políticos y la cultura.

\section{LOS JÓVENES Y LAS OPORTUNIDADES DE DESARROLLO EN SUS MUNICIPIOS}

Como ya se ha mencionado, los municipios de San Carlos y de San Francisco fueron duramente golpeados por el conflicto armado, lo que condujo a un atraso en el desarrollo de los territorios. Sin embargo, con los programas de desminado humanitario y el posterior retorno de las familias víctimas, se han creado planes que responden a las necesidades de la comunidad. Ahora bien, a pesar de estas políticas de desarrollo, los jóvenes han tenido expectativas distintas de las proyectadas por los municipios. Es decir, la tradición agrícola a la que sus padres estaban acostumbrados no representa para los jóvenes una cultura que se deba mantener, sino, por el contrario, sus proyectos de vida se encuentran en lugares distintos de sus territorios natales.

Esta situación se debe a las condiciones educativas, de participación, de construcción de ciudadanía, a las actividades económicas, a los medios de comunicación y a los programas sociales para jóvenes, que no son suficientes para la demanda juvenil. Anteriormente, se ha analizado el tema de la participación y es claro que los programas que 
sugieren los PDM de los municipios de San Carlos y de San Francisco no han tenido el impulso suficiente para vincular activamente a los jóvenes.

Las pocas oportunidades que brinda el municipio son algunas de las causas por las cuales los jóvenes se proyectan en otros lugares. En ambos territorios, predomina la población rural, por tanto, las oportunidades laborares se encuentran en el sector agropecuario, sin embargo, en las cabeceras municipales, también se desarrollan actividades económicas concentradas en el sector servicios, como educación, salud, servicios institucionales, comerciales, de recreación, etc. Entre estas, la de mayor productividad es la comercial, basada en la compra y venta de los productos que los campesinos traen de sus veredas, es decir, la vida socioeconómica gira en torno a lo rural.

No obstante, los jóvenes de territorios rurales han vivido transformaciones que los han obligado a dejar su identidad rural. Según Pérez (2001) y Gómez, Echeverri y Ribero (2002), citados por Jurado y Tobasura (2012), esas transformaciones obedecen a la diversificación de la estructura productiva, que ya no depende, exclusivamente, de las actividades agropecuarias; de la transformación de los estilos de vida y los valores que solían asociarse a lo rural, principalmente por el desarrollo de las tecnologías de la información y de la comunicación (TIC); y de la descentralización política, mediante la cual se busca darles mayor poder a las instancias locales y regionales.

La tablas 1 y 2 describen las oportunidades de educación superior en los municipios de San Francisco y de San Carlos.

Las tablas arrojan una diferencia visible en la oferta para cada municipio. Primero, el municipio de San Francisco tiene menos habitantes, de ahí que la oferta sea inferior; sin embargo, no se presentan muchas opciones de educación superior para los estudiantes, debido a que existen restricciones impuestas por la poca población interesada. Básicamente la oferta se encuentra en manos del SENA (MinTrabajo, 2013). Segundo, la oferta en el municipio de San Carlos es variada y permite que los bachilleres tengan la libertad de escoger entre varías carreras e instituciones educativas. Y tercero, la Secretaría de Educación sugiere que los programas ofertados sean oportunos en formación de actividades agropecuarias, para que no se

Tabla 1. Oferta de educación superior en el municipio de San Francisco

\begin{tabular}{|c|c|}
\hline \multirow{3}{*}{ SENA } & Técnica en Manejo Ambiental \\
\hline & Tecnología en Producción Agrícola \\
\hline & Técnica en Atención Integral a la Primera Infancia \\
\hline $\begin{array}{l}\text { Jóvenes con Futuro } \\
\text { (Gobernación Antioquia) }\end{array}$ & $\begin{array}{l}\text { Vincula jóvenes de } 16 \text { a } 29 \text { años en programas de formación } \\
\text { para el trabajo }\end{array}$ \\
\hline $\begin{array}{l}\text { Corporación de Estudios, Educación e } \\
\text { Investigación Ambiental (CEAM) }\end{array}$ & $\begin{array}{l}\text { Brinda alianzas con otras entidades para la asesoría en proyec- } \\
\text { tos agropecuarios, investigación y gestión de proyectos }\end{array}$ \\
\hline
\end{tabular}

Fuente: Elaboración propia según Ministerio de Trabajo (2013). 
Tabla 2. Oferta educación superior en el municipio de San Carlos

\begin{tabular}{|c|c|}
\hline \multirow{8}{*}{$\begin{array}{l}\text { Servicio Nacional de Aprendizaje } \\
\text { (SENA) }\end{array}$} & Tecnología Control de Ambiente \\
\hline & Tecnología Construcción \\
\hline & Técnica Mesa y Bar \\
\hline & Técnica Asistencia Administrativa \\
\hline & Técnica Producción Agropecuaria \\
\hline & Tecnología Gestión de Servicios Recreativos \\
\hline & Técnica Cocina \\
\hline & Tecnología Gestión de Recursos Naturales \\
\hline \multirow{3}{*}{ Universidad del Magdalena } & Tecnología en Educación Física \\
\hline & Tecnología en Salud Ocupacional \\
\hline & Licenciatura en Educación Preescolar \\
\hline $\begin{array}{l}\text { Corporación Unificada Nacional de Educación Supe- } \\
\text { rior }(\mathrm{CUN})\end{array}$ & Contaduría Pública \\
\hline \multirow{3}{*}{ Universidad Católica de Oriente } & Derecho \\
\hline & Zootecnia \\
\hline & Tecnología Agropecuaria \\
\hline Universidad Católica del Norte & Alfabetización de Adultos \\
\hline \multirow{2}{*}{ Escuela Superior de Administración Pública (ESAP) } & Tecnología Administración Pública Territorial \\
\hline & Tecnología Gestión Pública Ambiental \\
\hline Institución Universitaria Colegio Mayor de Antioquia & Tecnología Gestión Comunicativa \\
\hline
\end{tabular}

Fuente: Secretaría de Educación San Carlos (2014).

presenten problemas de relevo generacional en el sector productivo (MinTrabajo, 2014). Ante este panorama, los jóvenes del municipio de San Francisco tendrían más motivos para abandonar su territorio natal, teniendo en cuenta las escasas oportunidades que le brinda su municipio para desarrollar su proyecto de vida. El profesor Carlos Amaya de la Institución Educativa de San Francisco menciona que

el municipio no tiene oportunidades $\mathrm{u}$ ofertas para los profesionales del municipio por temas de recursos y la falta de empresas en la región. Además, escuchando a los muchachos de grado once, en su mayoría quieren partir a los grandes centros urbanos del país donde se encuentran las oportunidades. Así tengan la intención de regresar, que de hecho algunos sí la tienen, los desmotiva la baja calidad de vida del municipio comparada con la que ofrecen las ciudades. (comunicación personal, 18 de noviembre de 2017)

Ahora bien, en el municipio de San Carlos, sí cambia un poco la dinámica migratoria. Por un lado, los programas de participación han tenido mayor efecto, fruto de ello es que actualmente el municipio cuenta con un $\mathrm{CMJ}$, en el que reciben apoyo de la 
Fundación Mi Sangre y del SENA para la formación de líderes y construcción de la ciudadanía (Alcaldía de San Carlos, 2016). En cuanto a la educación, la oferta es mayor y diversa, además se ha intentado desarrollar un programa de recuperación de identidad con los jóvenes a través de la Casa de la Cultura a fin de reconstruir la memoria histórica del municipio.

Sin embargo, existe un problema muy arraigado entre la población del municipio de San Carlos, relacionado con la etiqueta "víctima de la violencia", que ha traído consigo la mano bondadosa del Estado para subsidiar a las personas que tuvieron que pasar por estos años de conflicto en el país. Es un problema en el sentido que "se creó una cultura de facilismo", como lo menciona el rector Jorge Lorenzo Sánchez, director del Institución Educativa Joaquín Cárdenas Gómez. Facilismo entendido como victimizarse para seguir obteniendo recursos del Estado. "Esta cultura ha impedido que los muchachos busquen otras alternativas de progreso, como es la educación, y prefieran estar amparados bajo el subsidio estatal" (comunicación personal, 17 de noviembre de 2017). Por tanto, la oferta educativa puede estar en el municipio, pero se necesita un acompañamiento educativo y cultural para incrementar el número de profesionales, así como mayores oportunidades laborales que ofrezcan a los jóvenes el desarrollo de un proyecto de vida dentro de su territorio natal.

\section{Consolidación de UNA ORGANIZACIÓN POLÍTICA JUVENIL}

A lo largo de este artículo, se ha mencionado la participación juvenil como necesaria para el desarrollo de los municipios, ya que contribuye a la inclusión social y a la construcción de la paz territorial. Su fundamento se encuentra en la sagrada norma colombiana, la Constitución Política de 1991, en la que se destaca el capítulo 3 del Régimen Municipal en su artículo 311, que establece:
Al municipio como entidad fundamen- tal de la división político-administrati- va del Estado le corresponde prestar los servicios públicos que determine la ley, construir las obras que demande el progreso local, ordenar el desarrollo de su territorio, promover la participación comunitaria, el mejoramiento social y cultural de sus habitantes y cumplir las demás funciones que le asignen la Constitución y las leyes.

La participación juvenil se define a través del Plan Municipal de Juventud (20162019), el cual reconoce a la juventud como sujeto de derechos y responsabilidades. Allí se propone como estrategia la implementación de la política pública de juventud y el fortalecimiento del CMJ. Sin embargo, en la realidad, no se está dando continuidad a las políticas ni a los mecanismos de participación juvenil. Por tanto, las alcaldías municipales deben brindar mayor atención a las juventudes por medio del acercamiento de las realidades propia de los jóvenes. Es decir, como ya se ha visto en los PDM de los municipios de San Carlos y de San Francisco, las políticas relacionadas con la juventud son ajenas a lo que ellos realmente quieren; tan solo basta recordar el contexto que acompaña su indiferencia y desapego por la organización y participación política. De modo que seguir con las mismas políticas de fortalecimiento tradicionales no va a 
generar ningún interés por parte de los jóvenes $y$, por tanto, van a seguir apareciendo propuestas inconclusas frente a la juventud.

Se trata más bien de un nuevo paradigma de participación juvenil, en el que las relaciones cotidianas permiten construir una nueva identidad. La propuesta es identificar la cultura como fundamento de la cohesión de la sociedad y la juventud, por medio del proyecto Conformación de colectivos de comunicación juvenil, lo cual implica un comportamiento social y político que, según Roseguillo (2014), son formas de participación juvenil no tradicionales.

Se entiende que los movimientos sociales eran fuertemente ideologizados y formalizados, con estructuras rígidas propias de juventudes políticas, movimientos estudiantiles clásicos, partidos obreros. En cambio, los colectivos juveniles actuales han sido caracterizados como más informales, aglutinados por formas horizontales y con "consignas" colectivas más directamente relacionadas con la vida cotidiana (vigencia de derechos sexuales y reproductivos, libertad de expresión a través de diversas manifestaciones culturales, etcétera). (Garcés, 2010, p. 66)

De acuerdo con Garcés (2010), los jóvenes en la actualidad se organizan en colectivos. Por eso, la Casa de la Cultura y de la Juventud son espacios fundamentales para que los jóvenes se reúnan y colectivamente expresen sus problemas frente a la sociedad. Desde allí se ejecuta el proyecto de Roseguillo Conformación de colectivos de comunicación juvenil. La realidad es que los jóvenes recurren a medios de protesta colectiva o individual, sin estar representados por una organización juvenil, de ahí que confíen en la movilización social como un medio para lograr intereses políticos, sociales y culturales. La transformación en las formas de organización juvenil ya no están asociadas a los movimientos formales y fuertemente estructurados, sino, por el contrario, la participación política juvenil evidencia que los jóvenes construyen redes informales para fines concretos e inmediatos. Es decir, la mejor forma de entender la participación juvenil es a través de las expresiones culturales juveniles, las cuales se entienden como un "conjunto heterogéneo de expresiones y prácticas socioculturales" (Martínez, 2008, p. 153), las cuales reflejan la relación que tienen los jóvenes con la sociedad. Es decir, las culturas juveniles van a ser la sustracción del análisis sociopolítico de la sociedad en la que se desarrollan.

Esta relación de la cultura y los jóvenes son una forma de participación alrededor de acciones y propuestas artísticas de resistencia, manifestadas a través de la música, la danza, el grafiti y otros medios de divulgación de divergencia política con alto impacto en el escenario público (Garcés, 2010). A estas manifestaciones alternativas, expresiones artísticas, interacciones con la tecnología y entre otras acciones juveniles se les conoce como "performatividad juvenil", es decir,

una sobre atención de las dimensiones tribales: códigos, emblemas, valores $\mathrm{y}$ representaciones que cohesionan al grupo, en detrimento de las dimensiones institucionales y del papel del mercado como rearticulador de los sentidos de pertenencia y ciudadanía $\mathrm{y}$, de manera especial, generaría una invisibilización analítica de lo que 
Chantal Mouffe ha denominado "los antagonismos políticos", el conflicto. (Reguillo, 2003, citado por Martínez, 2008, p. 153)

La performatividad o desdramatización juvenil se instala como un rechazo a la política tradicional y se presenta como una nueva forma de entender la participación política juvenil entre las diversas prácticas de los jóvenes. Por tanto, la política es un "estilo de vida estrechamente ligado a la cultura" (Reguillo, 2003). De ahí que los programas mencionados por el secretario de Gobierno del municipio de San Carlos referente a los murales sean tan importantes para explicar la forma en que los jóvenes participan en los municipios. Desde la Casa de la Cultura y la Juventud, se llevan a cabo prácticas juveniles que hablan de su relación con lo social y político. Este tipo de demostraciones son la materialización de los postulados de Reguillo, en los que se evidencia una ciudadanía juvenil estrechamente ligada a la cultura, ya que, al tener en cuenta otras esferas que van más ligadas a lo cotidiano, se restablece el tejido social, se participa y se crean territorios de paz. Es decir, en las prácticas del territorio, se desarrolla todo tipo de posibilidades para la participación juvenil.

\section{Conclusión}

La participación política juvenil en los municipios de San Carlos y de San Francisco ha sido relativamente baja desde el punto de vista de las formas tradicionales de participación. Entendiéndose formas tradicionales según el concepto de participación de Robert Dahl como la posibilidad que tienen los ciudadanos de incidir en los acontecimientos políticos a través del voto. Sin embargo, son otros los medios a través de los cuales los jóvenes participan. Al estudiar de manera detalla los PDM, se encontraron políticas que inciden en la participación de los jóvenes de estos municipios. De ahí que fuera necesario analizar la educación, la cultura y los programas de participación, con lo cual se concluyó que la educación es fundamental en la formación de los jóvenes y que, por tanto, el aumento de la calidad y la cobertura permitirían una conciencia crítica en los aspectos sociales y políticos que vive la sociedad. Asimismo, la cultura y los programas de participación que se encuentran dentro de los PDM dan cuenta de las falencias económicas y políticas para consolidar una participación masiva de los jóvenes.

Sin embargo, es de reconocer que el municipio de San Carlos, a diferencia del municipio de San Francisco, brinda mayor respaldo a las políticas de juventudes, a través de la Casa de la Cultura y de la Juventud. De hecho, gracias a los programas que allí se construyen surgen formas alternativas de participación por medio del arte, la música y el teatro. A esta participación se le conoce como perfomatividad juvenil, en la que las expresiones del cuerpo, la música, el arte y otras acciones juveniles tienen componentes sociales y políticos con los cuales se expresan las necesidades, las realidades y los deseos de la juventud en medio de la sociedad. Por medio de la perfomatividad juvenil, se lleva a cabo la construcción de la memoria histórica para que nunca en estos territorios se utilice la violencia como forma de expresión.

Los PDM contienen las políticas que dan paso a la participación juvenil a través de la creación de los consejos de juventudes 
y la implementación de la política pública de juventud, la cual reconoce la ciudadanía juvenil

más allá del voto o ejercicio formal de participación [...]. Visibiliza las características propias de la diversidad de los territorios, las formas de organización y participación de las juventudes y propone la concepción de la ciudadanía juvenil, como un ejercicio de relación y prácticas constantes y cotidianas de los y las jóvenes entre ellos y con otros actores sociales, políticos, económicos y culturales. (Ley Estatutaria 1622/2013, de 29 de abril)

Por tanto, la participación política juvenil se da a partir del reconocimiento de la ciudadanía, la cual dota de protección a los jóvenes a través de derechos y deberes.

No obstante, en los PDM no existe una continuidad en los pocos esfuerzos por las políticas juveniles. Más bien las políticas públicas de los municipios reflejan los intereses de las élites sin reflejar las demandas del pueblo. En este orden de ideas, los desafíos que se proponen para potencializar la eficacia de las políticas juveniles son:

- Control, veeduría y seguimiento a los planes, proyectos y políticas: esta estrategia va encaminada a divulgar las políticas que se desarrollan en las instituciones municipales y que los ciudadanos se empoderen de reclamar el seguimiento y la ejecución de los objetivos que se proponen.

- Inclusión dinámica para involucrar a los jóvenes: hacer uso de la tecnología para llegar a los jóvenes e incluirlos en la toma de decisiones de políticas públicas.

- Desarrollo de habilidades: reconocer las potencialidades de los jóvenes en los municipios e incluirlos como grupo empoderado dentro de la sociedad, respetando sus distintas formas de ver las realidades y expresándolas por medio de cultura juvenil.

Ahora bien, en relación con el acuerdo de paz, el punto dos brinda nuevas oportunidades a los municipios para integrar diferentes pensamientos frente a la participación política. Desde el acuerdo, se hace un llamado a cumplir con los deberes de participación ciudadana y se asegura que el Estado garantizará la protección de los movimientos y de las organizaciones sociales en los territorios. De ahí que los PDM actuales tengan como propósito la inclusión social, la cual se desarrolla a través de la participación de la comunidad en la construcción de propuestas conjuntas en pro de un mejor bienestar para todos, por medio de las JAC. Sin embargo, por más que existan políticas y en el acuerdo se exprese la intención por integrar a la juventud dentro del ámbito político, en la práctica el número de movimientos juveniles es reducido. Si bien el acuerdo abre la posibilidad de aumentar la inclusión social, hace falta mucho más compromiso en los programas juveniles, en especial con la memoria histórica, que permitirá construir territorios de paz para las generaciones futuras.

Los municipios de San Carlos y de San Francisco, tras ser golpeados por la violencia, vivieron grandes retrasos en economía y educación, los cuales afectan directamente a las nuevas generaciones, ya que, al finalizar el 
bachillerato, los jóvenes se sienten motivados a salir de sus municipios en busca de oportunidades en las grandes ciudades. En el municipio de San Francisco, la oferta en educación superior es inferior en comparación con la del municipio de San Carlos, pero los niveles en calidad institucional son más altos en el municipio de San Francisco. Por tanto, existe mayor expectativa en sus jóvenes por abandonar su municipio y acceder a la educación superior en otros lugares, con la esperanza de retornar, aunque lastimosamente el municipio no cuenta con la oferta laboral para brindar altas expectativas de vida a sus jóvenes dentro del territorio. Por otra parte, en el municipio de San Carlos, los jóvenes tienen mayor oferta educativa e incluso laboral (empresas hidroeléctricas, crecimiento del comercio y sector servicios), lo cual ha hecho que los jóvenes consideren como opción quedarse en sus territorios natales.

Finalmente, se puede concluir que la participación política juvenil en las políticas de desarrollo municipal ha sido marginal y poco incentivada, lo que ha impedido la consolidación de una organización juvenil. Por esta razón, los mismos jóvenes han creado sus propias manifestaciones de participación que se alejan de lo tradicional pero se acercan ampliamente a una ciudadanía cultural.

\section{REFERENCIAS}

"Acuerdo final para la terminación del conflicto y la construcción de una paz estable y duradera" (2016, noviembre 24). Recuperado de http://www.altocomisionadoparalapaz.gov.co/procesos-y-conversaciones/Documentos $\% 20$
compartidos/24-11-2016NuevoAcuerdoFinal.pdf

Alcaldía de San Carlos. (2012). Plan de Desarrollo Municipal (2012 -2015) "Unidos construyendo el San Carlos que queremos". San Carlos, Colombia: Autor.

Alcaldía San Carlos. (2016). Plan de Desarrollo Municipal (2016-2019) "Trabajando por el desarrollo social". San Carlos, Colombia: Autor.

Alcaldía de San Francisco. (2012). Plan de Desarrollo Municipal (2012-2015) "San Francisco con oportunidades para todos". San Francisco, Colombia: Autor.

Alcaldía de San Francisco. (2016). Plan de Desarrollo Municipal (2016-2019) "Unidos por el cambio de San Francisco". San Francisco, Colombia: Autor.

Booth, J. A. y Seligson, M. A. (1978). Political participation in latin America. Vol. 1: Citizen and state. Nueva York, EE. UU.: Holmes \& Meier.

Bourdieu, P. (1990). La juventud no es más que una palabra. En Autor, Sociología y cultura (pp. 163-173). Ciudad de México, México: Grijalbo.

Celis Ospina, J. C. (2014). Principales tendencias de la sociología del trabajo en Colombia entre 1993 y 2013. Revista Colombiana de Sociología, 37(1), 17-47.

Coordinación de Educación Municipal San Francisco Antioquia. (2015). Plan Educativo Municipal (2015-2023). San Francisco, Colombia: Alcaldía Municipal.

Constitución Política de Colombia. Recuperado de http://www.constitucioncolombia.com/

Cordourier Real, C. R. (2015). Participación ciudadana e instituciones: un análisis desde la teoría democrática de Robert A. Dahl. Estudios Sociológicos, 33(99), 579-605. 
Dahl, R. A. (1971/2013). La poliarquía: participación y oposición. Madrid, España: Tecnos.

Dastres, C. (2003). Reflexiones para la incorporación de la participación ciudadana en la gestión pública. Santiago de Chile, Chile: Universidad Central de Chile.

Delfino, G. I. y Zubieta, E. M. (2010). Participación política: concepto y modalidades. Anuario de Investigaciones, 17, 211-220.

Díaz Nagles, M. A. (2011). Cultura politica y juventud: el papel de la escuela en la formación política de los jóvenes. Bogotá, Colombia: Pontificia Universidad Javeriana.

Echeverría, J. (2009). Participación ciudadana y educación: ¿cómo la educación puede ampliar las posibilidades de participación? Polémika, 2(1), 26-37.

Garcés Montoya, Á. (2010). De organizaciones a colectivos juveniles: panorama de la participación política juvenil. Última Década, 18(32), 61-83.

Garzón Carrillo, R. J. (s. f.). Política y juventud: participación de los y las jóvenes en Fontibón. Recuperado de http://webiigg. sociales.uba.ar/iigg/jovenes_investigadores/5jornadasjovenes/EJE10/PonenciaGarzon.pdf

Gómez, J. D. (2009, octubre 3). Procedimiento para elaborar el Plan Educativo Municipal El Colombiano. Recuperado de http://www.elcolombiano. com/historico/procedimiento_para_ela borar_el_plan_educativo_municipal -DLEC 62274

Grupo de Memoria Histórica. (2011). San Carlos: memorias del éxodo en la guerra. Bogotá, Colombia: Autor.

Instituto Latinoamericano para una Sociedad y un Derecho Alternativos. (2009).
Desplazamiento forzado y retorno en San Carlos, Antioquia: una comunidad que regresa hacia el confinamiento. Bogotá, Colombia: Autor.

Jurado Alvarán, C. y Tobasura Acuña, I. (2012). Dilema de la juventud en territorios rurales de Colombia: ¿campo o ciudad? Revista Latinoamericana de Ciencias Sociales, Niñez y Juventud, 10(1), 63-77.

Ley 152/1994, de 15 de julio, por la cual se establece la Ley Orgánica del Plan de Desarrollo.

Ley Estatutaria 1622/2013, de 29 de abril, por medio de la cual se expide el estatuto de ciudadanía juvenil y se dictan otras disposiciones.

Martínez, J. E. (2008). Participación política juvenil como políticas del acontecimiento. Revista Argentina de Sociología, 6(11), 148-168.

Ministerio de Trabajo. (2013). Perfil productivo municipio de San Francisco. Bogotá: Autor.

Ministerio de Trabajo. (2014). Perfil productivo municipio de San Carlos. Bogotá: Autor.

Murillo, T. J. (2006). Cuestionarios y escalas de actitudes. Madrid, España: Universidad Autónoma de Madrid.

Reguillo, R. (2003a). Ciudadanías juveniles en América Latina. Última Década, 11(19), 11-30.

Reguillo, R. (2003b). Las culturas juveniles. Un campo de estudio: breve agenda para la discusión. Revista Brasileira de Educação, 23, 103-118.

Reguillo, R. (2004). La performatividad de las culturas juveniles. Revista de Estudios de Juventud, 64, 49-56.

Secretaría de Educación San Carlos Antioquia. (2011). Plan Educativo Municipal (2012-2021). San Carlos, Colombia: Alcaldía Municipal. 
Somuano Ventura, M. F. (2005). Más allá del voto: modos de participación política no electoral en México. Foro Internacional, 45(1), 65-88.

Tamayo Arango, A. S. (2017). El dolor habla: memoria histórica de las mujeres víctimas del conflicto armado del municipio de San Francisco, Antioquia. Medellín, Colombia: Universidad Católica Luis Amigó.
Varón Cárdenas, D. (2014). La cuidadanía juvenil y los mecanismos de participación en jóvenes: el Estatuto de Cuidadanía Juvenil (Ley 1622 de 2013) y la construcción de la realidad desde el derecho. Verba Iuris, 31, 115-134.

Verba, S. y Nie. H. N. (1972). Participation in America: Political democracy and social equality. Nueva York, EE. UU.: Harper and Row. 